\title{
Narrativas sobre el proceso de recuperación ante la adicción: la perspectiva de familiares que asisten a servicios de atención
}

\author{
Narratives about the process of recovering from \\ addiction: the perspective of family members attending \\ care services
}

${ }^{1}$ Autor de correspondencia. Doctor en Psicología. Profesor-Investigador, Universidad Autónoma de Baja California, Mexicali, México. $\square$ iD

${ }^{2}$ Doctora en Psicología. Profesora-Investigadora Universidad Autónoma de Baja California, Mexicali, México. $\square$ iD
RESUMEN Los servicios de tratamiento para las adicciones suelen priorizar la recuperación del usuario, a pesar de que el consumo problemático implica un malestar en las familias que requiere atención. El objetivo de este estudio es identificar los tipos de narrativas que construyen los familiares de personas con trastornos por dependencia a sustancias, acerca del padecimiento y el proceso de recuperación, así como comprender las condiciones que facilitan u obstaculizan el proceso de recuperación desde la perspectiva de los familiares. Se realizaron siete entrevistas en profundidad con orientación narrativa. Se encontraron narrativas progresivas, estables y regresivas en las que se identificaron diversas etapas en los procesos de adicción y recuperación, y revelaron diferentes formas de malestar, barreras para el tratamiento, y momentos críticos para asistir a los servicios de atención. Las familias representan un recurso valioso para la recuperación del usuario, por lo que deben ser incluidas en el tratamiento.

PALABRAS CLAVES Narrativa Personal; Recuperación de la Salud Mental; Trastornos Relacionados con Sustancias; Relaciones Familiares; México.

\footnotetext{
ABSTRACT Addiction treatment services usually prioritize the recovery of the user, despite the fact that problematic consumption often leads to distress among families that requires attention. The purpose of this study is to identify types of narratives constructed by relatives of people with substance dependence disorders regarding their condition and recovery process. Additionally, we seek to understand the conditions that facilitate or hinder the recovery process from the perspective of family members. Seven in-depth interviews were conducted with a narrative approach. Progressive, stable, and regressive narratives were identified, corresponding to various stages in the addiction and recovery processes. These narratives revealed different forms of distress, obstacles to treatment, and critical junctures with respect to attending care services. Families represent a valuable resource for user recovery, and therefore must be included in the treatment process.

KEY WORDS Personal Narrative; Mental Health Recovery; Substance-Related Disorders; Family Relationships; Mexico.
} 


\section{INTRODUCCIÓN}

En México, el 2,2\% de la población presenta dependencia al consumo de alcohol y el $0,6 \%$ a las sustancias ilícitas. Un 13,9\% de las personas con dependencia al alcohol y el $20,4 \%$ de las personas con dependencia a sustancias ilícitas han acudido a tratamiento. Los varones presentan un mayor consumo de ambos tipos de sustancias y asisten con mayor frecuencia al tratamiento ${ }^{(1,2)}$. Antes de asistir a los servicios de atención especializados en adicciones, los usuarios suelen recurrir a sus familiares y amistades, a la automedicación o las instituciones religiosas, lo cual contribuye a la progresión del padecimiento y el aumento de su severidad ${ }^{(3)}$.

Existen tres principales fuentes de servicios de tratamiento. La primera, es el sector público con más de 400 centros de cuidado y aproximadamente 30 unidades de tratamiento residencial. La segunda fuente son los servicios profesionales de salud privados $y$, la tercera, está conformada por grupos de autoayuda tradicionales, con aproximadamente 20.000 grupos alrededor del país y 2.291 centros de ayuda mutua residenciales, conocidos popularmente como "anexos"(4).

La bibliografía reporta que la familia influye en el desarrollo de la adicción. Los usuarios pueden haber estado inmersos en un triángulo relacional, en el que se sobreinvolucran con uno de los padres y se distancian o están en conflicto con el otro; estos conflictos en el sistema limitan el establecimiento de límites consistentes. Otra razón por la cual no se establecen límites es por el temor a que uno de sus miembros pierda el control, de modo que el usuario aprende a actuar en el mundo bajo la premisa básica de que el contexto social no le impone restricciones ${ }^{(5)}$. Otros autores han considerado que el consumo de drogas es parte de un patrón relacional que permite mantener la estabilidad de la familia y desviar el conflicto, además de que es una manifestación de una seudoindividuación del usuario por el miedo a la separación experimentado por la familia ${ }^{(6)}$. A su vez, se ha señalado que existen múltiples pautas o recorridos a través de los cuales se desarrolla la conducta adictiva, que implican la transmisión generacional de carencias afectivas, la participación dentro de un juego relacional donde se sobreinvolucra con uno de los padres y se distancia del otro, y la conducta adictiva se convierte en una forma de buscar autonomía y lidiar con el malestar producido por el abandono parental ${ }^{(7)}$.

Los servicios de tratamiento han priorizado la recuperación individual del usuario, a pesar de que el consumo problemático de sustancias lícitas e ilícitas implica una carga en los familiares que requiere ser reconocida $y$ atendida ${ }^{(8)}$. Estudios previos indican que genera diversas consecuencias en los miembros de la familia, tales como incertidumbre, preocupación, ambivalencia, malestar emocional y físico; conflictos y dilemas asociados al manejo del usuario; oscilación entre diversas estrategias de afrontamiento; problemas económicos; restricción de la vida social, vergüenza, aislamiento; y disminución en la calidad de las relaciones familiares ${ }^{(9,10)}$. A pesar de ello, en México son escasas las intervenciones que se han enfocado en las necesidades de familiares de personas que abusan de sustancias ${ }^{(11)}$.

La inclusión de miembros de la familia en los servicios especializados en adicciones no solo es importante para la atención de sus necesidades, sino porque pueden convertirse en un recurso valioso para el tratamiento al promover el inicio del proceso de recuperación ${ }^{(12,13)}$. Junto con el internamiento voluntario, el internamiento propuesto por los familiares es uno de los motivos más reportados para ingresar a los centros residenciales de ayuda en México ${ }^{(4)}$. Estudios previos recomiendan incorporar a los familiares en los tratamientos porque la recuperación es un proceso dinámico que no solo ocurre a través del tiempo, sino que se relaciona con redes de personas de forma similar a la adicción ${ }^{(14,15)}$; y durante el tratamiento, pueden facilitar la recuperación por medio de la retroalimentación al usuario, el apoyo instrumental y emocional, o convertirse en motivaciones para el cambio ${ }^{(16)}$. La participación de los familiares no solo favorece el ingreso del usuario al tratamiento, 
sino que aumenta las probabilidades de que lo concluya y obtenga mejores resultados ${ }^{(17,18,19)}$.

Las intervenciones que involucran a familiares pueden agruparse en tres categorías: intervenciones con miembros de la familia para promover la entrada y la participación de los usuarios en el tratamiento, participación conjunta de miembros de la familia y usuarios en el tratamiento de estos, e intervenciones que responden a las necesidades de los familiares ${ }^{(20)}$. La psicoeducación, el apoyo por parte de los familiares y el desarrollo de habilidades de afrontamiento son elementos esenciales de las intervenciones con familias ${ }^{(21)}$.

Entre los tratamientos que contemplan la participación conjunta del usuario y su familia se encuentra la terapia familiar multidimensional ${ }^{(22)}$, un tratamiento integral para adolescentes con problemas de conducta y consumo de drogas, basado en la familia y orientado a múltiples sistemas (usuario, padres, familia y sistemas más amplios); así como la terapia familiar multidimensional con perspectiva de género ${ }^{(23)}$, dirigida a la atención del consumo de drogas y la violencia familiar. Por otra parte, los tratamientos para atender las necesidades de los familiares se enfocan en reducir el estrés y aumentar las habilidades de afrontamiento ${ }^{(24)}$; desarrollar estas mismas habilidades en familias indígenas para que aprendan a lidiar con el consumo excesivo de alcohol(11); $y$, en general, apoyar a familias con problemas por el consumo de alcohol y drogas ${ }^{(25)}$.

A pesar de que la familia es uno de los contextos donde puede identificarse en forma temprana el consumo problemático de sustancias, existe una serie de barreras que limitan la búsqueda de apoyo en los servicios de atención por parte de los miembros. Social y culturalmente se considera el consumo de sustancias como una cuestión privada que debe ser resuelta por la misma familia, y se responsabiliza principalmente a las mujeres por el consumo de sus miembros, lo cual puede ser complejo para las mujeres, considerando que múltiples celebraciones y eventos sociales suelen ir acompañadas del consumo de sustancias legales, principalmente el alcohol y tabaco, promoviéndose de esta forma cierta tolerancia social del consumo. Asimismo, existen comunidades en las que el consumo de sustancias legales, como el alcohol, no es considerado un problema. En contextos de pobreza y marginación, por ejemplo, existen otras necesidades familiares a las que se les brinda prioridad antes que al tratamiento.

El aplazamiento de la internación se relaciona con el deseo de manejar el asunto en forma privada debido al estigma asociado a las adicciones, de fomentar la tolerancia del consumo del familiar, de normalizar el consumo de sustancias lícitas como el alcohol(26), así como de identificar y vincular el problema de consumo con una cuestión moral que depende de la voluntad del usuario o del apoyo exclusivo de la familia ${ }^{(27)}$. De forma análoga a lo que ocurre con el usuario, los miembros de la familia acuden inicialmente a formas no especializadas de apoyo, como la familia extensa o las instituciones religiosas. Posteriormente, pueden recurrir a profesionales de la salud mental como psicólogos o psiquiatras, en especial, del sector privado. Finalmente, una barrera importante es que la adicción puede ser concebida en un inicio como un "vicio", por lo que se teme que el usuario sea excluido de los servicios de salud si se asume públicamente como "adicto". Al concebirse la adicción como un problema moral, se asume que la fuerza de voluntad es el factor único o central para erradicar la adicción ${ }^{(27)}$.

A propósito del término adicción, cabe señalar que en este estudio se le otorga el sentido de una experiencia factible de narrarse. Las narrativas que el usuario y su familia cuentan representan narrativas de aflicción que dejan al descubierto trayectorias de personas, creencias, categorías e instituciones, que muestran la variedad de situaciones que existen detrás de la etiqueta de la adicción ${ }^{(28)}$.

\section{Narrar el padecimiento}

Padecimientos como el VIH y las adicciones suelen ir acompañados de procesos de estigma y discriminación que suponen distintos tipos de rupturas (de la realidad cotidiana, de la identidad y biográficas), que conllevan a una nueva construcción de significados 
relacionados consigo mismo, con los otros, con el padecimiento y con la recuperación ${ }^{(29)}$. Para comprender la forma en que los familiares participan en el proceso de recuperación de usuarios de sustancias lícitas e ilícitas, se pueden explorar sus narrativas; es decir, aquellos relatos que dan cuenta del proceso mediante el cual los familiares organizan la trayectoria de adicción y recuperación, y enfatizan aquellos aspectos que dan sentido a su participación en dicho proceso.

Desde una perspectiva socioconstruccionista, la narrativa del padecimiento es la historia que el paciente cuenta, mientras los familiares y amigos re-cuentan esa misma historia pero desde su propia perspectiva, para dar coherencia a los eventos distintivos y al curso del sufrimiento a largo plazo ${ }^{(30)}$. Las narrativas no solo permiten construir un mundo del padecimiento, sino también reconstruir la narrativa de la historia de vida, la explicación y comprensión del padecimiento, la justificación de las propias acciones ante el otro, así como trasladar la experiencia del padecimiento de la esfera privada hacia sus implicaciones sociales ${ }^{(31)}$. Además, la acción de relatar moldea la forma en que se viven los síntomas, el tratamiento y el sufrimiento generado por el padecimiento e influye en la postura que se asume ante este, pues al narrar se puede abrir el futuro a un final positivo e imaginar medios de superar la adversidad ${ }^{(32)}$.

Los usuarios y sus familias construyen estrategias narrativas en el intento de situar el sufrimiento en la historia personal y organizar los acontecimientos relacionados con el padecimiento en un orden significativo a través del tiempo ${ }^{(32)}$. Se pueden diferenciar tres tipos generales de narrativas. Las narrativas progresivas se caracterizan porque se incrementa la evaluación de los acontecimientos a través del tiempo, pues se avanza en las dificultades y se anticipa que su dirección continúe de forma ascendente, como en los géneros de la épica o la comedia. En las narrativas regresivas ocurre una evaluación progresivamente decreciente, la situación empeora y se anticipa que su dirección prosiga de forma descendente, como en el género de la tragedia; mientras que, en las narrativas estables, la trayectoria del relato no se altera en relación con la meta o resultado, la dirección se mantiene, ya sea en un sentido de evaluación positiva o negativa ${ }^{(33)}$.

Frank $^{(34)}$ ha propuesto una tipología de narrativas en pacientes que sufren un padecimiento. Un primer tipo son las narrativas de restitución, que inician con un sufrimiento físico y la falla social, seguido del encuentro con un remedio o tratamiento, y la restauración de la comodidad física y obligaciones sociales gracias a la agencia del personal de salud. Un segundo tipo son las narrativas caóticas, centradas en el presente y sin una secuencia, pues el paciente es avasallado no solo por la sintomatología del padecimiento, sino por otras dificultades sociales y económicas. Estas narrativas refieren a la pérdida del control, la vulnerabilidad y los límites del tratamiento. El tercer tipo son las narrativas de búsqueda, que inician con el sufrimiento generado por el padecimiento y culminan con un sentido construido a partir de la experiencia del padecimiento. El paciente se vuelve agente, y su relato puede funcionar como una memoria de su vida, denuncia social o mostrar su transformación personal(34).

El propósito del presente estudio es identificar los tipos de narrativas que construyen los familiares de usuarios diagnosticados con trastornos por dependencia a sustancias lícitas o ilícitas, acerca del padecimiento y el proceso de recuperación. Asimismo, se busca comprender las condiciones que facilitan u obstaculizan el proceso de recuperación, desde la perspectiva de los familiares que brindan acompañamiento durante el tratamiento.

\section{METODOLOGÍA}

El estudio se realizó en una clínica de internación y rehabilitación para las adicciones, una comunidad terapéutica que opera con el modelo Minnesota ${ }^{(35)}$ y pertenece al sector público en Ciudad Victoria, localizada en la región central del estado de Tamaulipas, que se encuentra en el noreste de México, en la frontera con Texas, EEUU. En esta región, la incidencia acumulada del uso de cualquier 
droga y de drogas ilegales en la población de 12 a 65 años ha disminuido entre el año 2008 y 2016, hasta alcanzar niveles cercanos al promedio nacional ${ }^{(1,2)}$. Además de las barreras de acceso al tratamiento mencionadas previamente, en Tamaulipas existen barreras contextuales ligadas al narcotráfico y la violencia. Tanto en centros de atención primaria como de internación, el personal de salud, los usuarios y sus familias carecen de condiciones de seguridad. En la comunidad y en los centros de atención han Ilegado a sufrir amenazas, desapariciones, riñas, o atentados directos contra los usuarios de sustancias, o el personal que trabaja allí(36).

En un primer momento se acudió a la dirección de la institución con el fin de presentar el proyecto y obtener la autorización para su realización. En la clínica se promovió que los investigadores contactaran inicialmente al personal de salud (psicólogos, psiquiatras, enfermeras, nutriólogos y personas con experiencia de adicción y recuperación que dirigen los grupos de autoayuda). Por medio de ellos se realizó la invitación a los familiares (personas relacionadas con el usuario por vínculos emocionales y parentesco de sangre o legal), quienes han vivido el proceso de adicción y participan una vez a la semana en grupos de autoayuda. Se realizaron entrevistas en profundidad, que fueron audio-grabadas y transcritas para su análisis. Las entrevistas fueron realizadas por la segunda autora de este trabajo, quien en ese momento trabajaba como investigadora en una institución de educación superior, por lo que no tenía relación alguna con el personal de salud, los usuarios de sustancias o los familiares de estos. Las entrevistas se realizaron en sesiones programadas, en un periodo de tres meses.

La orientación metodológica empleada fue narrativa ${ }^{(33)}$, pues se buscó comprender la trayectoria desde el inicio del consumo hasta el momento actual del proceso de recuperación.

Los participantes fueron seleccionados en forma propositiva, de acuerdo a los siguientes criterios: ser adultos, que fueran familiares de un usuario que haya sido diagnosticado con un trastorno por dependencia a sustancias, y que ellos y/o el usuario se encontraran en proceso de tratamiento. En el estudio participaron siete familiares, de los cuales, cinco de las participantes eran mujeres y dos varones, principalmente, eran esposas o madres. Tenían un rango de edad de 23 a 51 años, con diversas ocupaciones: ama de casa, estudiante, comerciante, empleado,

Tabla 1. Características relacionadas con el consumo de los usuarios que asisten a centro de rehabilitación en Tamaulipas, México.

\begin{tabular}{|c|c|c|c|c|c|c|c|}
\hline Caso & $\begin{array}{l}\text { Parentesco } \\
\text { del familiar } \\
\text { participante }\end{array}$ & $\begin{array}{l}\text { Sexo del } \\
\text { usuario }\end{array}$ & $\begin{array}{c}\text { Edad del } \\
\text { usuario }\end{array}$ & $\begin{array}{l}\text { Sustancias } \\
\text { consumidas }\end{array}$ & $\begin{array}{l}\text { Tiempo de } \\
\text { consumo } \\
\text { (años) }\end{array}$ & $\begin{array}{l}\text { Tiempo en } \\
\text { recuperación } \\
\text { (años) }\end{array}$ & $\begin{array}{l}\text { Número de veces } \\
\text { en que ha sido } \\
\text { internado }\end{array}$ \\
\hline 1 & Esposa & Hombre & 51 & Alcohol, morfina & 22 & 2 & 5 \\
\hline 2 & Esposa & Hombre & 56 & Alcohol & 9 & 23 & $0^{*}$ \\
\hline 3 & Mamá & Mujer & 19 & $\begin{array}{l}\text { Marihuana, } \\
\text { LSD, alcohol }\end{array}$ & 3 & 1 & 2 \\
\hline 4 & Mamá & Hombre & 23 & $\begin{array}{l}\text { Marihuana, } \\
\text { solventes }\end{array}$ & 1 & 2 & 2 \\
\hline 5 & Tío & Hombre & 43 & Alcohol & 10 & 0 & 0 \\
\hline 6 & Esposa & Hombre & 50 & $\begin{array}{c}\text { Alcohol, } \\
\text { medicamentos } \\
\text { fuera de } \\
\text { prescripción }\end{array}$ & 28 & 2 & 1 \\
\hline 7 & Papá & Hombre & 20 & $\begin{array}{c}\text { Alcohol, } \\
\text { marihuana, } \\
\text { cocaína, crack }\end{array}$ & 4 & 2 & 4 \\
\hline
\end{tabular}


funcionario gubernamental o consejero en adicciones. La mayoría estaban casadas, y solo dos se encontraban en situación de separación o divorcio. En la Tabla 1 se presentan las características de los usuarios de sustancias que los participantes acompañaban al centro de tratamiento.

Para el desarrollo de las entrevistas se empleó la siguiente pregunta generadora: “ ¿me puede contar la historia desde que su familiar empezó a consumir hasta el momento actual del tratamiento?". Dentro de la etapa de consumo, se abordaron los siguientes temas: inicio del consumo, creencias y afectos hacia el consumo, acciones ante el consumo, consecuencias del consumo, indicadores del agravamiento del consumo, y relación con las redes sociales de apoyo y los servicios de atención. En la etapa del tratamiento, se exploraron los motivos de las internaciones y las acciones para facilitarlas; las creencias y afectos hacia la recuperación; la participación en el tratamiento y los cambios percibidos durante ese proceso.

Las entrevistas tuvieron una duración de una a tres horas. Inicialmente, se realizó un análisis categórico de las narrativas para la identificación de creencias, afectos, prácticas e interacciones de los familiares con instancias externas durante el proceso de adicción y de recuperación. Posteriormente, se empleó un análisis holístico de las narrativas para identificar el tipo de narrativa empleada en cada caso y las fases por las que transitaban los familiares ${ }^{(37)}$. Dichas fases expresan periodos en los que la adicción se vive de cierta forma. El paso de una fase a otra demarca cambios significativos en las trayectorias de adicción y recuperación. Finalmente, se emplearon las categorías previamente identificadas para comprender los elementos que facilitan u obstaculizan el proceso de recuperación, en cada una de las fases.

A los participantes se les explicó el objetivo de la investigación y se les entregó un consentimiento informado. El protocolo de investigación fue aprobado por el Comité de Ética de la Investigación del Cuerpo Académico "Migración, desarrollo y derechos humanos", perteneciente a la Universidad Autónoma de Tamaulipas.

\section{RESULTADOS}

\section{Narrativas progresivas: recuperación del usuario y sus familiares}

La recuperación se refiere al proceso en el que los usuarios y sus familiares recobran lo que perdieron durante el proceso de adicción, como la salud mental, física y emocional; los vínculos sanos, las redes sociales, el trabajo, entre otros; además de incluir un periodo de abstinencia o de consumo no problemático de sustancias. Se denominaron como narrativas progresivas aquellas en las que se mostraba que la recuperación del usuario y su familia se había extendido temporalmente, e incluso podía indicarse que ocurría una transformación en el usuario o el familiar. Se identificaron tres tipos de narrativas progresivas.

En la narrativa progresiva principal, los cambios sucedieron, en un primer momento, en los familiares, quienes promovieron la internación y los cambios posteriores del usuario. Corresponde a los casos uno y tres, en los que los usuarios presentan policonsumo y los familiares se identifican como codependientes. La fase inicial de esta narrativa se denominó "normalización del consumo", pues este no es percibido como problemático cuando no se ha detectado el consumo de sustancias ilícitas, se conserva el funcionamiento social y económico del usuario, y existe una identificación positiva al ser buen proveedor o no ejercer violencia. Aunque esta fase se relata en forma breve, puede tener varios años de duración.

\footnotetext{
Pues transcurría entre lo normal y lo anormal, pero para mí ya era normal, sus salidas muy frecuentes a tomar, a perderse, a veces se hundía, a veces mucho más. (Esposa, caso 1)
}

La segunda fase se llamó "sufrimiento por el consumo". Se caracteriza porque el usuario aumentaba la frecuencia del consumo o empezaba a consumir sustancias ilícitas, principalmente con amistades y de forma oculta. Las familiares comenzaban a percibir un 
deterioro psicosocial, pues notaban alteraciones del "carácter", problemas de salud, educativos o laborales, o que desaparecía durante varios días. Otras consecuencias eran los problemas económicos, los conflictos con el usuario y entre los miembros de la familia, incluyendo el maltrato hacia los hijos. Además, se producía una sobrecarga en el familiar respecto al cuidado del hogar y problemas de salud. Al interior de la familia existían dificultades para establecer límites ante el consumo y desacuerdo para su manejo.

Eso fue peor cada vez lo de la tomada porque siempre tomaba él, pero como quiera estaba presente en días pues importantes o días que lo necesitaba. Pero con la tercera, nació y al otro día que salí del hospital, él se fue disque a Tampico, para arreglar el seguro de gastos del hospital y se me desapareció más de 15 días. Entonces todo eso fue muy duro. (Esposa, caso 1)

Las dificultades anteriores favorecen que el consumo sea percibido como problemático y se genere ambivalencia hacia el usuario. Las familiares recurían a diversas estrategias ante el problema, como escapar o distraerse, vivir para los hijos, expresar el malestar al usuario o intentos de mantener estable la vida cotidiana y acostumbrarse a la situación. Las familiares podían acudir a un tratamiento psicológico en esta fase, pero predominaba la búsqueda de apoyo personal en la familia extensa o en la comunidad religiosa, pues el usuario no asistía a los servicios de atención. La familia extensa podía involucrarse negativamente, ya sea culpando al familiar, o promoviendo que acepte el consumo. Además, las familiares experimentaban la ausencia de una guía para el manejo del problema, lo cual se asociaba con la falta de visibilidad de los servicios de atención.

La tercera fase se denominó "cambio en el manejo del consumo". Corresponde a cuando el familiar, quien asume un papel más activo como agente en el proceso de recuperación, "toca fondo". Este escenario se favorecía no solo por el agravamiento del consumo, sino porque ocurrían problemas laborales o escolares detectados por instancias externas, así como eventos críticos como el intento de suicidio o la desaparición por lapsos extensos de tiempo, que originaban un cambio en los afectos de las familiares hacia el usuario, y el predominio de diversas expresiones de malestar, como desconexión, coraje [ira], desconfianza, vergüenza, tristeza, o preocupación. Además, se modificaba la percepción del familiar sobre el problema, al asumir que el usuario no podría cambiar por sí mismo y que no eran suficientes las promesas de cambio o el apoyo de la familia, sino que requería de atención especializada. Por ello, comenzaba un proceso de peregrinaje en busca de la institución adecuada, que podía variar en función de la visibilidad de los servicios de atención y también de su calidad. Una manifestación de la discriminación hacia los usuarios de drogas es la ausencia de servicios de calidad y que se habiliten centros de tratamiento donde se violan sus derechos humanos.

En esta fase las familiares pueden asistir a terapia individual o grupos de familias, pero a la vez promueven que el usuario asista a atención psiquiátrica, intervenciones familiares o se interne en un centro residencial. Usualmente no es suficiente el acompañamiento a estos servicios, sino que requieren establecer límites para forzarlo a asistir. Cabe señalar que las familias no suelen acudir al primer nivel de atención, sino directamente a los centros de rehabilitación. El cambio parece ocurrir en forma gradual y discontinua, es decir, implica una sucesión de internamientos y recaídas, en las que el usuario vuelve a consumir sustancias después de un periodo de abstinencia. Las internaciones también se convierten en mecanismos de control cuando las familias ya no pueden manejar el consumo del usuario por su cuenta.

La última fase se denominó "mantenerse en la institución adecuada". El usuario se estabilizaba, asistía en forma continua al tratamiento, y adoptaba la ideología del tratamiento promovida por la institución, por lo que el consumo se construía como una enfermedad principalmente psicológica y se asumía que era necesario un tratamiento 
intensivo y crónico, lo que implicaba un cambio de vida y que la familia se involucrara en la recuperación. Las familiares se identificaban como codependientes y reconocían haber llegado a sentirse responsables del consumo, sobre todo cuando eran mujeres y percibían cambios positivos en el usuario. En forma paralela al usuario convertido en paciente, asistía a grupos para familias o a sesiones familiares, y adoptaban ciertas prácticas terapéuticas en la vida cotidiana, como conocer su propia enfermedad y analizar el papel de la familia de origen en su inicio, así como "no cargar cosas de los demás". En esta fase se percibían cambios en el usuario como reconectarse con la familia, ser responsable o alejarse de amistades que consumían. En la familia ocurrían cambios en relación con la expresión de emociones hacia el usuario y el establecimiento de límites, la reducción del conflicto y el incremento de la cercanía emocional, mejoras en la comunicación, la incorporación del usuario a las tareas del hogar, además de que la familiar se enfocaba en cambiar aspectos negativos de su persona, lo que hacía que el usuario se sintiera menos solo. Se expresaba un sentido de crecimiento y transformación tras el sufrimiento, y ocurría un cambio en la red social, pues se incorporaba el personal de salud y las familias de otros usuarios.

\begin{abstract}
Aquí fue donde se me dijo que yo tenía una enfermedad que se llamaba la codependencia. De hecho, mi mamá vive fuera de aquí, como a 40 kilómetros de la ciudad... ni siquiera me iba dos tres días, porque yo pensaba que, si me iba, algo malo le iba a pasar. Como que me sentía el amuleto de él ¿verdad? O sea, estando yo en casa con mis hijos, él va a llegar. Y si yo me voy, o sea, algo malo le va a pasar. (Mamá, caso 3)
\end{abstract}

Un segundo tipo de narrativa progresiva corresponde al caso dos, en el que el usuario acude a atención por consumo de alcohol. En esta narrativa el cambio individual del usuario precede a la transformación de la familia. Tal como ocurre en la narrativa previa, existe una fase de "sufrimiento por el consumo", pero se diferencia de la siguiente fase de "ingresar a Alcohólicos Anónimos". En este relato el usuario aceptaba el problema de consumo, acudía voluntariamente a un grupo de Alcohólicos Anónimos sin tener que ser internado, y se mantenía en recuperación con escaso involucramiento de la familia en el tratamiento, al menos en un inicio. Posteriormente, la familiar adoptó la ideología del tratamiento: consideraba que el consumo es producto de una enfermedad y de una personalidad adictiva, se identificaba como codependiente y realizaba las prácticas terapéuticas promovidas por el programa de 12 pasos.

\section{Yo llegué al grupo por la adicción de mi marido, pero me di cuenta que el pro- blema no es la adicción, no es la sustan- cia... yo cuando les explico les digo, vas a hacer un duelo, a la sustancia, pero no a la adicción, la adicción está en ti, el alcohol es el alcohol y no te hace daño, la marihuana es la marihuana, es una planta, las pastillas... si son médicas son para un tratamiento, pero a ti te hacen daño, te hace daño cuando entra a tu cuerpo, entonces la enfermedad está en ti, ¿qué es la enfermedad? La obsesión. (Esposa, caso 2)}

Si bien se interioriza la ideología del tratamiento y ocurre una transformación personal en el usuario y la familiar, esa narrativa tiene una última fase que no aparece en el resto de los relatos, denominada "una nueva vida". En esta fase ambos miembros de la familia se han convertido en consejeros y ayudan a otros pacientes en recuperación, pero también refieren otros asuntos vitales no relacionados con el consumo, como la creación de un negocio, lidiar con dificultades económicas y promover un crecimiento en la relación de pareja (Tabla 2).

El tercer tipo de narrativa progresiva, corresponde al caso seis, en el que el usuario presentaba policonsumo, tenía una sola internación y su familiar no se identificaba como codependiente. En esta narrativa también ocurrían cambios en el usuario y en el 
Tabla 2. Tipos de narrativas progresivas construidas por familiares de usuarios que asisten a centro de rehabilitación en Tamaulipas, México.

\begin{tabular}{|c|c|c|c|c|}
\hline Tipo de narrativa & \multicolumn{4}{|c|}{ Etapas } \\
\hline $\begin{array}{l}\text { Progresiva I: } \\
\text { cambios en usuario, } \\
\text { luego en familiares }\end{array}$ & - & Sufrimiento por el consumo & $\begin{array}{l}\text { Ingresar a Alcohólicos } \\
\text { Anónimos }\end{array}$ & Una nueva vida \\
\hline $\begin{array}{l}\text { Sustancia de } \\
\text { impacto: } \\
\text { alcohol }\end{array}$ & - & $\begin{array}{l}\text { Consecuencias del consumo } \\
\text { en ámbito económico, laboral } \\
\text { y familiar } \\
\text { Malestar emocional y } \\
\text { ambivalencia hacia el usuario } \\
\text { El consumo se considera como } \\
\text { un problema, que puede ser } \\
\text { manejado en la familia } \\
\text { Se recurre a fuentes no } \\
\text { especializadas de apoyo: } \\
\text { familia extensa y religión }\end{array}$ & $\begin{array}{l}\text { El usuario tiene una epifanía, } \\
\text { y asiste voluntariamente al } \\
\text { grupo } \\
\text { Familiar del usuario adopta } \\
\text { ideología del tratamiento } \\
\text { y se identifica como co- } \\
\text { dependiente } \\
\text { Cambios en el consumo, } \\
\text { carácter, relaciones familiares } \\
\text { y funcionamiento social } \\
\text { (paciente y familiar) }\end{array}$ & $\begin{array}{l}\text { Interiorizar la ideología del } \\
\text { tratamiento } \\
\text { Convertirse en consejeros y } \\
\text { ayudar a otros } \\
\text { Enfocarse en otras cuestiones } \\
\text { vitales, como dificultades } \\
\text { económicas, crear un negocio } 0 \\
\text { asuntos de pareja } \\
\text { Se otorga un sentido de } \\
\text { crecimiento tras el sufrimiento }\end{array}$ \\
\hline $\begin{array}{l}\text { Progresiva II: } \\
\text { cambios en } \\
\text { familiares, luego en } \\
\text { usuario }\end{array}$ & Normalización del consumo & Sufrimiento por el consumo & $\begin{array}{l}\text { Cambio en el manejo del } \\
\text { consumo }\end{array}$ & $\begin{array}{l}\text { Mantenerse en la institución } \\
\text { adecuada }\end{array}$ \\
\hline $\begin{array}{l}\text { Policonsumo } \\
\text { (alcohol + cocaína } \\
\text { + morfina; alcohol } \\
\text { + marihuana) }\end{array}$ & $\begin{array}{l}\text { Consumo no se percibe como } \\
\text { problemático. } \\
\text { No se consumen o no se } \\
\text { ha detectado consumo de } \\
\text { sustancias ilícitas. } \\
\text { Imagen positiva del usuario; } \\
\text { mantenimiento de función } \\
\text { social y económica. }\end{array}$ & $\begin{array}{l}\text { Aumento del consumo y del } \\
\text { deterioro psicosocial } \\
\text { Malestar emocional y } \\
\text { ambivalencia hacia el usuario } \\
\text { El consumo se considera como } \\
\text { un problema, que puede ser } \\
\text { manejado en la familia } \\
\text { Se recurre a fuentes no } \\
\text { especializadas (familia extensa } \\
\text { y religión) y especializadas } \\
\text { (terapia individual) de apoyo }\end{array}$ & $\begin{array}{l}\text { Familiar "toca fondo" } \\
\text { Intensificación del deterioro y } \\
\text { eventos críticos } \\
\text { Intensificación del malestar } \\
\text { Se percibe que el usuario no } \\
\text { podrá cambiar por sí mismo y } \\
\text { requiere ayuda especializada } \\
\text { Deambular en busca de } \\
\text { tratamiento adecuado } \\
\text { Sucesión de internamientos } \\
\text { y recaídas }\end{array}$ & $\begin{array}{l}\text { Estabilización del paciente y } \\
\text { asistencia al tratamiento } \\
\text { Familiar adopta ideología del } \\
\text { tratamiento y se identifica } \\
\text { como codependiente } \\
\text { Asisten a intervenciones para } \\
\text { familiares y adoptan prácticas } \\
\text { terapéuticas en la cotidianidad } \\
\text { Cambios en el consumo, } \\
\text { relaciones familiares y } \\
\text { funcionamiento social; } \\
\text { se percibe un proceso de } \\
\text { transformación }\end{array}$ \\
\hline
\end{tabular}

Fuente: elaboración propia.

familiar, pero los cambios eran más abruptos y se desarrollaban en un marco temporal más breve. La fase inicial se nombró "atender la crisis". Antes de la crisis, el consumo de alcohol del usuario no era considerado problemático y era percibido como alguien funcional pues contaba con un empleo, era responsable y buen proveedor. En comparación con los relatos anteriores, no se presentaba una fase extensa de sufrimiento por la adicción ni un peregrinaje en busca del tratamiento adecuado. Ocurría un evento crítico, el intento de suicidio del usuario en un momento de su vida en el que comenzaba a consumir benzodiacepinas sin prescripción médica y se entrelazaban diversos acontecimientos importantes, como la pérdida de un negocio, la muerte de su madre y el cuidado de su padre por enfermedad. La familia experimentaba malestar emocional, pero se movilizaba rápidamente ante la crisis, y recibía principalmente servicios especializados como una intervención tanatológica, terapia familiar e individual, y atención psiquiátrica para el usuario. También recibían apoyo de la familia extensa y de la comunidad religiosa. La esposa del usuario estableció límites para favorecer que el usuario acudiera a la internación.

O sea, él vino aquí bien, ni con engaños no lo trajimos, así como que amarrado ni nada... por voluntad, aja, pero si como a sabiendas, como quiera, que si no vas a ayuda pues, o sea, yo me voy con mi hija. (Esposa, caso 6)

En la segunda fase, "mantenerse en tratamiento", ocurrieron procesos similares a las narrativas anteriores. La diferencia es que en este 
caso la internación fue inmediata a la crisis, los familiares acompañaban al usuario en el tratamiento y asistían a las actividades del centro de rehabilitación, pero no se identificaban como codependientes. Se reconocieron cambios en el consumo, en el "carácter" del usuario y en las relaciones familiares, pero no se otorgaba un sentido de transformación personal o de crecimiento como en los relatos previos.

\section{Narrativas estables: cambios en el familiar, pero no en el usuario}

Esta narrativa corresponde al caso cinco, en el que el usuario consumía alcohol, y no asistía a tratamiento. La primera fase se denominó "vida con el consumo del usuario". En comparación con las narrativas progresivas, no había una fase de sufrimiento asociado al consumo ni la presencia de una crisis. Entre las consecuencias principales del consumo se encontraban los conflictos familiares con el usuario. En la familia se reconocía que había un problema, pero se acostumbraron a él. El familiar, en este caso varón, señalaba que, al haberse enfocado en el consumo del usuario, descuidó sus propias relaciones y su salud. Su búsqueda de tratamiento no se debía únicamente al consumo del usuario, sino a que padeció depresión, en un periodo de su vida en el que lidió con sucesos vitales estresantes como la separación de su pareja y un accidente automovilístico.

Cuando yo empecé a buscar ayuda por la depresión, me doy cuenta que traía arrastrando ciertos daños por convivir con un alcohólico. (Tío, caso 5)

La segunda fase del relato fue llamada "seguir en tratamiento para la co-dependencia". A pesar de que el usuario seguía consumiendo sin acudir a tratamiento, el familiar adoptaba la ideología de cuidado que considera que la adicción es una enfermedad y que el usuario va a asistir cuando "toque fondo". Se identificaba como codependiente, asistía a grupos para familiares y en su vida cotidiana adoptaba prácticas promovidas por la filosofía de los doce pasos: reconocer la influencia de la familia de origen en el problema, responsabilizarse de sus propias acciones, compartir en el grupo, honestidad, manejar las emociones, "no cargar las cosas de otros", decidir lo que quiere para sí mismo, ser humilde, hacer un inventario, buscar el perdón, ayudar a otros que pasan por un problema similar, conocerse a sí mismo para comprender la enfermedad, y relacionarse con un poder superior. Tal como ocurre en narrativas progresivas, expresaba un sentido de crecimiento a partir del tratamiento, pero no se manifestaban indicadores de intensificación del malestar a través de la trayectoria del relato, tampoco indicios de recuperación por parte del usuario (Tabla 3 ).

\section{Narrativas regresivas: en espera de la recuperación}

Las narrativas regresivas se caracterizan por el predominio de un sentido trágico en el relato, ya sea porque el usuario no ha logrado recuperarse o la recuperación es parcial y de duración breve, con el riesgo latente de la próxima recaída. Se encontraron dos tipos de narrativas regresivas. En el primer tipo, existía un consumo problemático de sustancias a través de varias generaciones y el familiar se identificaba como "adicto en recuperación". Este relato corresponde al caso cuatro, en el que el usuario presenta policonsumo y ha vivido múltiples internaciones.

La primera fase de la narrativa se denominó "llevar una vida de sufrimiento". La madre del usuario narraba sobre su propia niñez y adolescencia, haciendo referencia al consumo problemático de alcohol por parte de su padre, la vivencia de maltrato, tener que trabajar desde niña e irse a vivir con otros familiares por la situación en el hogar. La familiar empezó a consumir alcohol desde la adolescencia, vivió en situación de calle, tuvo que recurrir al sexo para supervivencia, y tuvo varios hijos con diferentes hombres que no la apoyaron en la crianza ni económicamente. Luego, se mudó para reunirse con su familia de origen y trabajar en el negocio 
Tabla 3. Tipos de narrativas progresivas y estables construidas por familiares de usuarios que asisten a centro de rehabilitación en Tamaulipas, México.

\begin{tabular}{|c|c|c|}
\hline Tipo de narrativa & & Etapas \\
\hline $\begin{array}{l}\text { Progresiva III: } \\
\text { cambios en ambos, tras } \\
\text { crisis }\end{array}$ & Atender la crisis & Mantenerse en tratamiento \\
\hline $\begin{array}{l}\text { Policonsumo } \\
\text { (alcohol + benzodiacepinas) }\end{array}$ & $\begin{array}{l}\text { No se problematiza el consumo previo; el usuario era } \\
\text { considerado funcional } \\
\text { Evento crítico que favorece el internamiento (intento de } \\
\text { suicidio) } \\
\text { Intensificación del malestar emocional } \\
\text { Principalmente acuden a servicios especializados } \\
\text { de atención (terapia individual y familiar, consulta } \\
\text { psiquiátrica) } \\
\text { Familiar establece límites para promover el internamiento }\end{array}$ & $\begin{array}{l}\text { Inicio inmediato del internamiento tras crisis } \\
\text { Paciente acude a cuidado continuo en el centro y a grupo de } \\
\text { Alcohólicos Anónimos } \\
\text { Familiar asiste a intervenciones familiares y realiza prácticas } \\
\text { terapéuticas en la cotidianidad } \\
\text { Adopción parcial de la ideología del tratamiento, familiar no se } \\
\text { identifica como codependiente } \\
\text { Cambios en el consumo, carácter y relaciones familiares } \\
\text { No se otorga un sentido de transformación personal tras la crisis }\end{array}$ \\
\hline $\begin{array}{l}\text { Estable: } \\
\text { cambios en el familiar, } \\
\text { no en el usuario (sin } \\
\text { intensificación del } \\
\text { deterioro) }\end{array}$ & Vida con el consumo del usuario & Seguir en tratamiento para la codependencia \\
\hline $\begin{array}{l}\text { Sustancia de impacto: } \\
\text { alcohol }\end{array}$ & $\begin{array}{l}\text { Se reconoce la presencia de un problema, pero se } \\
\text { acostumbra } \\
\text { Se presentan manifestaciones leves del sufrimiento, no } \\
\text { aparecen crisis asociadas al consumo o indicadores de } \\
\text { grave deterioro psicosocial del usuario } \\
\text { El familiar tiene una epifanía tras distintos sucesos vitales } \\
\text { que promueven la búsqueda de atención }\end{array}$ & $\begin{array}{l}\text { El familiar acude a grupos para familiares de usuarios y se } \\
\text { identifica como codependiente. } \\
\text { Adopta la ideología del tratamiento y sigue sus prácticas } \\
\text { terapéuticas en la vida cotidiana } \\
\text { Otorga un sentido de transformación personal } \\
\text { El usuario continúa el consumo y no se encuentra en tratamiento. } \\
\text { El familiar espera que lo haga hasta que "toque fondo" }\end{array}$ \\
\hline
\end{tabular}

Fuente: Elaboración propia

familiar. Decidió vivir por su propia cuenta con sus hijos varones, seguía consumiendo y tuvo una nueva pareja estable que también consumía. En esta fase del relato no se refería al consumo de su hijo, sino a su propia historia personal como preámbulo para el problema del usuario.

La segunda fase se denominó "darse cuenta del consumo". La familiar tardó en identificar que su hijo tiene un problema de consumo. No reconocía las señales de alerta como problemas escolares y tampoco atendía las recomendaciones del personal escolar de procurar atención especializada. La madre reconocía el problema de consumo de sus hijos en la adolescencia, pero existían conflictos familiares y sentimientos de desconexión, ira, ambivalencia, desconfianza y preocupación. Se generaban diversas posturas respecto al manejo del consumo, por lo que la madre oscilaba entre establecer límites y no mantenerlos. Se recurría en menor medida a redes informales de apoyo, como la comunidad religiosa y la familia extensa. La madre intentaba recuperarse regresando al grupo de Alcohólicos Anónimos, aunque no se comprometía con el tratamiento. Por su parte, el hijo ingresaba a una primera internación en un centro de rehabilitación. El proceso de recuperación de ambos se vio obstaculizado por un evento crítico en la familia: el otro hijo fue asesinado. Esto ocurrió tras un periodo de consumo de drogas, una sucesión de internaciones, y no tener un sitio donde vivir en un contexto con alto nivel de violencia asociada al crimen organizado.

La tercera fase se denominó "intentos de recuperación". En esta fase la madre se identificaba como "adicta en recuperación" y consideraba que requería atenderse para acompañar a su hijo en el tratamiento, quien se había internado de manera voluntaria. La madre asistía a su propio grupo de Alcohólicos Anónimos, así como a las sesiones familiares o grupos de 
familias que organizaba el centro de rehabilitación. La madre consideraba el consumo como una enfermedad y adoptaba prácticas basadas en la filosofía de los doce pasos. La madre identificaba cambios en sí misma en términos de responsabilidad, dejar de consumir y alejarse de las amistades que consumen, vincularse afectivamente con su hijo, mejorar su comunicación e involucrarlo en el hogar. En esta fase la madre había desarrollado un compromiso con su propia recuperación, pero el usuario continuaba internado, en un nuevo intento por recuperarse. Entre las barreras que limitan este proceso se perciben: la adaptación al "encierro", la disponibilidad de sustancias en la vida cotidiana, los conflictos familiares y la falta de compromiso personal con el tratamiento.

\section{Cuando me dijo: "quiero internarme, ya no quiero drogarme", entonces ahí fue donde, me dice el padrino, "empieza a creer, ya te dio la prueba". Y es cuando empiezo yo realmente a creer tantito, poquitito ¿verdad? Cuando ya empieza mi hijo a ingresar aquí a la clínica y todo eso y empezarme a pegar más al grupo, pero eso realmente tiene poquitito que me acabo de dar cuenta. Casi voy a hacer 4 años en el grupo y no me había dado cuenta yo de eso. (Madre, caso 4)}

El segundo tipo de narrativa regresiva se caracteriza porque no se reporta la recuperación del usuario ni del familiar. Este relato es narrado por el padre y corresponde al caso siete, en el que el usuario presentaba policonsumo y había tenido múltiples internaciones. En este relato no se reporta una historia de consumo en varias generaciones, sino que inicia en la fase de "darse cuenta del consumo". La identificación es tardía porque el usuario abandonó el hogar tempranamente por un embarazo adolescente y el consumo se mantenía oculto. Detectaron el consumo por múltiples consecuencias psicosociales: desaparecer por días, conflictos de pareja y cambios de hogar, robo para consumir, alteraciones en el "carácter", problemas para mantener un trabajo y en su salud. Como se ha mencionado en otros relatos, en la familia predominaban sentimientos de ira, desconfianza y desconexión con el usuario. Existían posturas encontradas respecto al manejo del consumo, pero gradualmente establecían límites para promover la internación. Se enfrentaban a la falta de información sobre tratamientos especializados y al estigma hacia los usuarios, que se manifestaba en la calidad de servicios disponibles en el sector público. Hubo varias internaciones por las recaídas, así como cambios en los centros de atención.

Él se enojaba con la mujer y regresaba a la casa, y luego después se volvía a ir y así estaba. Y una vez, le agarró, a mi yerno la tarjeta. Entonces fue ahí cuando nosotros nos dimos cuenta de que él era el autor de las cosas que se perdían. Y le preguntamos cuál era el detalle. ¿Sabes qué? Ahorita te voy a entregar a la policía y ya fue cuando él dijo "Es que yo ya no puedo" ¿Por qué no puedes? ¿Qué tienes o qué? "Pues es que yo... yo soy adicto". (Papá, caso 7)

La segunda fase se denominó "en espera de la recuperación". Los familiares acompañaban al usuario en una nueva internación, pero no había indicios de progreso, el consumo se había cronificado y había aumentado el deterioro psicosocial. Predominaba el malestar y la identificación negativa del usuario, se mantenía la incertidumbre por una nueva recaída. El padre asistía a sesiones familiares o grupos para familias en el centro de rehabilitación, pero no se identificaba como codependiente ni adoptaba prácticas basadas en la ideología del tratamiento. No reportaba cambios en sí mismo ni el usuario, tampoco había una transformación del sentido otorgado a la vida con el consumo. Como barreras para la recuperación del usuario, se perciben: la tentación de consumir, los conflictos con la familia, la disponibilidad de sustancias, contar con redes sociales en las que se consume, así como el maltrato recibido tanto en centros de rehabilitación como centros de ayuda mutua residenciales (Tabla 4 ). 
Tabla 4. Tipos de narrativas regresivas construidas por familiares de usuarios que asisten a centro de rehabilitación en Tamaulipas, México.

\begin{tabular}{|c|c|c|c|}
\hline Tipo de narrativa & & Etapas & \\
\hline $\begin{array}{l}\text { Regresiva I: } \\
\text { no hay cambios en el usuario, el } \\
\text { familiar está en recuperación }\end{array}$ & $\begin{array}{l}\text { Llevar una vida de } \\
\text { sufrimiento }\end{array}$ & Darse cuenta del consumo & Intentos de recuperación \\
\hline $\begin{array}{l}\text { Policonsumo } \\
\text { (alcohol + marihuana + } \\
\text { inhalables) }\end{array}$ & $\begin{array}{l}\text { Familiar se enfoca en } \\
\text { su propia historia de } \\
\text { sufrimiento (abuso de } \\
\text { alcohol del padre, maltrato, } \\
\text { conflictos con la familia, vivir } \\
\text { en situación de calle y sexo } \\
\text { por supervivencia, tener } \\
\text { hijos con múltiples parejas). } \\
\text { El consumo problemático se } \\
\text { inicia en la adolescencia y se } \\
\text { mantiene en la etapa adulta. } \\
\text { Dificultades para la crianza, } \\
\text { continuación de conflictos } \\
\text { con la familia de origen y } \\
\text { falta de redes de apoyo. }\end{array}$ & $\begin{array}{l}\text { Identificación del consumo de sus hijos en } \\
\text { la adolescencia. } \\
\text { Continuación del consumo de la madre, } \\
\text { no hay compromiso con su propio } \\
\text { tratamiento. } \\
\text { Malestar por el consumo del hijo y } \\
\text { dificultades para el establecer límites ante } \\
\text { el consumo. } \\
\text { El hijo es internado en un centro y recae. } \\
\text { Evento crítico: otro de sus hijos es } \\
\text { asesinado (consumía drogas, vivía en } \\
\text { distintos lugares, había salido y entrado } \\
\text { de centros de rehabilitación). }\end{array}$ & $\begin{array}{l}\text { La madre se identifica como "adicta en } \\
\text { recuperación", acude a grupo de Alcohólicos } \\
\text { Anónimos. } \\
\text { El hijo acepta internarse voluntariamente y } \\
\text { ella acompaña al hijo en intervenciones para } \\
\text { familiares, promovidas por el centro. } \\
\text { La madre adopta la ideología del } \\
\text { tratamiento y realiza las prácticas } \\
\text { propuestas en la vida cotidiana. } \\
\text { La madre identifica cambios en sí misma en } \\
\text { términos de dejar de consumir, cambiar sus } \\
\text { relaciones sociales, ser más responsable y en } \\
\text { el vínculo con su hijo. } \\
\text { El hijo sigue en tratamiento, pero se } \\
\text { encuentran en espera de cambios } \\
\text { significativos. }\end{array}$ \\
\hline $\begin{array}{l}\text { Regresiva II: } \\
\text { no hay cambios en el familiar ni } \\
\text { en el usuario }\end{array}$ & - & Darse cuenta del consumo & En espera de la recuperación \\
\hline $\begin{array}{l}\text { Policonsumo } \\
\text { (alcohol + marihuana + cocaína } \\
+ \text { crack) }\end{array}$ & - & $\begin{array}{l}\text { Además de que el usuario no vive con la } \\
\text { familia, el consumo se oculta y se detecta } \\
\text { tardíamente el problema, hasta que se } \\
\text { vuelve evidente el deterioro psicosocial. } \\
\text { Malestar emocional por el consumo y } \\
\text { posturas encontradas sobre su manejo. } \\
\text { Deambular en busca de tratamiento; } \\
\text { sucesión de internamientos y recaídas. }\end{array}$ & $\begin{array}{l}\text { Ocurre un nuevo internamiento, pero no hay } \\
\text { indicios de progreso y se teme una nueva } \\
\text { recaída. } \\
\text { Predomina malestar emocional e } \\
\text { identificación negativa del usuario. } \\
\text { El familiar asiste a sesiones para familias } \\
\text { en el centro, pero no se identifica como } \\
\text { codependiente ni adopta prácticas } \\
\text { terapéuticas cotidianas. } \\
\text { No se reportan cambios en el familiar ni en } \\
\text { el usuario. }\end{array}$ \\
\hline
\end{tabular}

Fuente: Elaboración propia.

\section{DISCUSIÓN Y CONCLUSIONES}

Antes de acudir a los servicios de atención, los usuarios recurren a la autoatención y a miembros de su red social cercana, como los familiares ${ }^{(3)}$. Si bien en el estudio se halló que los miembros de la familia, promovían la internación de los usuarios ${ }^{(4)}$, este proceso podía retrasarse, lo cual contribuía a la cronificación del problema de consumo.

Un hallazgo del estudio es que los familiares o los usuarios en forma individual no solían acudir a los centros de primer nivel de atención cuando el consumo se tornaba problemático. Posiblemente esto se relacione con el estigma hacia las adicciones, pues acudir a un centro de atención puede implicar reconocerse públicamente como "adicto", lo cual está ligado al "vicio" desde el discurso moral. Aunque también es posible que se deba a una desvinculación de los centros de atención con la comunidad, ya que los familiares no contaban con información suficiente sobre las alternativas de tratamiento. Las principales opciones empleadas por los participantes fueron los grupos de Alcohólicos Anónimos y los "anexos" o centros de ayuda mutua residenciales. Se ha señalado que estos últimos no solo presentan deficiencias en la calidad de los servicios de atención, sino que además se cometen violaciones a los derechos humanos 
de los usuarios ${ }^{(38)}$. La falta de acceso a un servicio de atención digno es una manifestación de la discriminación hacia los usuarios de sustancias, la cual contribuye a que el problema de consumo se extienda en el tiempo y se agrave el deterioro psicosocial.

Tal como se ha encontrado en estudios $\operatorname{previos}^{(9,10)}$, los miembros de la familia experimentan diversas formas de malestar emocional, como ira, tristeza, preocupación, desconfianza y ambivalencia. Ocurren conflictos con el usuario y entre los miembros, además de distanciamiento, rechazo e identificación negativa. El deterioro psicosocial del usuario puede afectar su capacidad para seguir trabajando, lo cual deriva en problemas económicos, junto con los gastos generados por el padecimiento. Asimismo, los miembros de la familia pueden desarrollar problemas de salud física y mental.

A pesar de la carga generada por el padecimiento, los familiares no suelen recibir servicios de atención dirigidos exclusivamente a ellos ${ }^{(11)}$. En el presente estudio se identificaron otras formas de atención, como los grupos de familiares y la incorporación de los familiares en el tratamiento del usuario. Otra manera de acceder a servicios de atención dirigidos al familiar es cuando este se identifica como codependiente, lo cual ocurre con mayor frecuencia en mujeres, dado que existe una diferenciación por género del cuidado de los usuarios. La utilización de la etiqueta diagnóstica de "codependiente" por parte de los servicios de salud requiere cuestionarse si contribuye a la patologización de un comportamiento promovido en las mujeres en su contexto sociocultural ${ }^{(39)}$, y si además favorece la exclusión de otros miembros de la familia del tratamiento, como los varones, los niños y los adolescentes.

Respecto a las narrativas, se identificaron distintos tipos. Dentro de las narrativas progresivas, se encontraron narrativas de búsqueda que acentúan el proceso de transformación personal(34). Estas pueden ser continuas, con un papel central del usuario como agente, o discontinuas, con periodos regresivos de sufrimiento y oscilaciones entre internaciones y recaídas. A su vez, se encontró una narrativa progresiva de restitución, que no se enfocaba en la transformación personal, sino en la recuperación del usuario y la familia tras una crisis, gracias al tratamiento en la clínica de rehabilitación. La narrativa de restitución se caracteriza por una temporalidad más breve, un cambio súbito y continuo a mayor velocidad. Las narrativas progresivas y estables identificadas en el estudio son similares a ciertos tipos de narrativas encontradas previamente con relación al proceso de recuperación: las narrativas de Alcohólicos Anónimos, crecimiento y codependencia ${ }^{(40)}$. En cambio, los relatos regresivos se caracterizan por la persistencia del malestar y de la incertidumbre ante la recaída, por una falta de confianza en el tratamiento y por la no adopción plena de su ideología. La recuperación se encuentra en espera, y el usuario se encuentra en una situación de mayor deterioro psicosocial. Son relatos en los que puede existir una historia de consumo por varias generaciones, violencia de género y violencia estructural ${ }^{(41)}$.

Si bien el presente estudio no busca una patologización de los miembros de la familia, entre los elementos que facilitan la cronificación del consumo se encuentran las dificultades en el establecimiento de límites, la oscilación entre diversas estrategias de manejo del consumo y los desacuerdos entre los miembros de la familia sobre el abordaje del problema. Adicionalmente, la minimización o normalización del consumo, y mantener el problema dentro de los confines del espacio familiar restringen las posibilidades de obtener apoyo en sistemas externos a la familia.

La recuperación se facilita cuando los miembros de la familia "tocan fondo". Este momento requiere que el familiar reconozca su propio malestar emocional y considere que ha alcanzado un límite, sobre todo, favorecido por la ocurrencia de eventos críticos o la amplificación del deterioro psicosocial del usuario y la carga generada por el padecimiento. No se trata únicamente de un proceso afectivo, sino también simbólico, ya que implica un cambio en la problematización del consumo, es decir, se reconoce que no se trata únicamente de un vicio, que no depende únicamente de 
la voluntad del usuario o de la familia, y que requiere de atención especializada. Si bien la aceptación del problema por parte del usuario y la internación voluntario pueden agilizar el proceso, los familiares también pueden promover que se establezcan ciertos límites. Además de la detección temprana e intervención oportuna ante el problema de consumo, es necesario señalar que las familias requieren alternativas de atención, que sean visibles en su comunidad y que brinden tratamiento de calidad para evitar el peregrinaje en busca de la institución apropiada.

El contacto con los servicios de atención supone otro cambio importante: pasar del abordaje familiar del problema, al abordaje no institucionalizado por medio de las redes de apoyo informales, hasta los servicios especializados en adicciones. En este momento, será fundamental que la familia no solo acompañe al usuario en el tratamiento, sino que reciban atención para poder cambiar las pautas relacionales que han mantenido con el usuario y la forma en que responden al consumo o manejan las recaídas ${ }^{(42)}$. Esto se facilita con la adopción de la ideología del cuidado del centro de rehabilitación ${ }^{(43,44)}$.
Aunque existen narrativas en las que las epifanías o eventos críticos facilitan el cambio súbito y continuo, en los relatos se encuentra que el cambio es principalmente discontinuo, que incluye una sucesión de periodos de sufrimiento, internaciones y recaídas, que gradualmente facilitan el proceso de recuperación ${ }^{(45)}$. Se encuentra que existen diversos escenarios de la recuperación: se recuperan ambos, se recupera la familia pero no el usuario, no se recupera ninguno o se recupera el usuario pero no la familia. Este último escenario es hipotético, pues no se identificó en los relatos analizados. Asimismo, puede ocurrir el cambio en distintas direcciones, ya sea que el usuario asuma un papel de agente y promueva la recuperación de la familia, o que los miembros de la familia asuman una mayor agencia y fomenten el cambio en el usuario. Los servicios de atención pueden recurrir a la familia como un recurso en la identificación temprana, atención oportuna y acompañamiento en la recuperación, así como para que sus integrantes accedan a servicios especialmente dirigidos a ellos, dad la carga generada por el padecimiento a través del tiempo.

\section{REFERENCIAS BIBLIOGRÁFICAS}

1. Instituto Nacional de Psiquiatría Ramón de la Fuente Muñiz, Instituto Nacional de Salud Pública, Comisión Nacional contra las Adicciones, Secretaría de Salud. Encuesta Nacional de Consumo de Drogas, Alcohol y Tabaco 2016-2017: Reporte de Alcohol [Internet]. México: INPRFM; 2017 [citado 26 ago 2019]. Disponible en: https://tinyurl.com/y9zzygv3.

2. Instituto Nacional de Psiquiatría Ramón de la Fuente Muñiz, Instituto Nacional de Salud Pública, Comisión Nacional contra las Adicciones, Secretaría de Salud. Encuesta Nacional de Consumo de Drogas, Alcohol y Tabaco 2016-2017: Reporte de Drogas [Internet]. México: INPRFM; 2017 [citado 26 ago 2019]. Disponible en: https://tinyurl.com/y7vbylru.

3. Berenzon S, Medina-Mora ME, Lara MA. Servicios de salud mental: veinticinco años de investigación. Salud Mental. 2003;26(5):61-72.
4. Marín-Navarrete R, Medina-Mora ME, Tena-Suck A. Addiction care in Mexico: a challenge for non-specialized health professionals. Salud Mental. 2016;39(5):241-242.

5. Schwartzman J. Addict abstinence and the illusion of alternatives. Ethos. 1977;5(2):138-150.

6. Stanton MD, Todd TC, Heard DB, Kirschner S, Kleinman JI, Mowatt DT, Riley P, Scott SM, Van Deusen JM. Un modelo conceptual. En: Stanton MD, Todd TC. Terapia familiar del abuso y adicción a las drogas. Barcelona: Gedisa; 1985. p. 25-42.

7. Cirillo S, Berrini R, Cambiaso G, Mazza R. La familia del toxicodependiente. Barcelona: Paidós; 1999.

8. Selbekk AS, Sagvaag $H$, Fauske $H$. Addiction, families and treatment: a critical realist search for theories that can improve practice. Addiction Research \& Theory. 2014;23(3): 196-204. doi: 10.3109/16066359. 2014.954555. 
9. Orford J, Velleman R, Copello A, Templeton L, Ibanga $\mathrm{A}$. The experiences of affected family members: a summary of two decades of qualitative research. Drugs: Education, Prevention and Policy. 2010;17(Suppl 1):44-62. doi: 10.3109/09687637.2010.514192.

10. Orford J, Velleman R, Natera G, Templeton L, Copello $\mathrm{A}$. Addiction in the family is a major but neglected contributor to the global burden of adult ill-health. Social Science \& Medicine. 2013;78(2):70-77. doi: 10.1016/j. socscimed.2012.11.036.

11. Natera G, Tiburcio M. Tailoring an intervention model to help indigenous families cope with excesive drinking in central Mexico. Salud Mental. 2007;30(6):32 42.

12. Madsen $\mathrm{P}$, Marsden-Hughes $\mathrm{H}$. Exploring the processes involved in long-term recovery from chronic alcohol addiction within an abstinence-based model: implications for practice. Counselling and Psychotherapy Research. 2013;13(3):201-209. doi: 10.1080/14733145. 2012.733716.

13. Wagner V, Bertrand K, Flores-Aranda J, Acier D, Brunelle $N$, Landry $M$, Brochu S. Initiation of addiction treatment and access to services: young adults' accounts of their help-seeking experiences. Qualitative Health Research. 2016;27(11):1614-1627. doi: $10.1177 / 1049732316679372$.

14. Wisely C. The logic of recovery and injection drug use. Journal of Substance Use. 2013;18(1):56-64. doi: 10.3109/14659891.2013.761024.

15. Candil A. Acompañar a usuarios intensivos de drogas: el papel de las redes de proximidad en los tratamientos ambulatorios. Antípoda, Revista de Antropología y Arqueología. 2016;26:179-196.

16. Brown S, Tracy EM, Jun M, Park H, Min M. Personal network recovery enablers and relapse risks for women with substance dependence. Qualitative Health Research. 2015;25(3):371-385. doi: 10.1177/1049732314551055.

17. Edwards ME, Steinglass P. Family therapy treatment outcomes for alcoholism. Journal of Marital and Family Therapy. 1995;21(4):475-509. doi: 10.1111/j.1752-0606. 1995.tb00176.x.

18. Epstein EE, McCrady BS. Behavioral couples treatment of alcohol and drug use disorders: current status and innovations. Clinical Psychology Review. 1998;18(6):689711. doi: 10.1016/s0272-7358(98)00025-7.

19. Meads C, Ting S, Dretzke J, Bayliss S. A systematic review of the clinical and cost-effectiveness of psychological therapy involving family and friends in alcohol misuse or dependence. Birmingham: University of Birmingham, Department of Public Health and Epidemiology; 2007.

20. Copello A, Velleman R, Templeton L. Family interventions in the treatment of alcohol and drug problems. Drug and Alcohol Review. 2005;24(4):369-385. doi: 10.1080/09595230500302356.
21. Lucksted A, McFarlane W, Downing D, Dixon L. Recent developments in family psychoeducation as an evidence-based practice. Journal of Marital and Family Therapy. 2012;38(1):101-121. doi: 10.1111/j.17520606.2011.00256.x.

22. Liddle HA, (ed.). Multidimensional family therapy for adolescent cannabis users, Cannabis Youth Treatment Series, Volume 5. Rockville: Center for Substance Abuse Treatment, Substance Abuse and Mental Health Service Administration; 2002.

23. Fernández C, González J, Barrera, G. Modelo de terapia familiar multidimensional con perspectiva de género. México: Centros de Integración Juvenil; 2006.

24. Orford J, Copello A, Velleman R, Templeton, L. Family members affected by a close relative's addiction: the stress-strain-coping-support model. Drugs: Education, Prevention and Policy. 2010;17(Suppl 1):S36S43. doi: 10.3109/09687637.2010.514801.

25. Natera G, Tiburcio M, Mora J, Orford J. Apoyando a familias que enfrentan problemas por el consumo de alcohol y drogas. México: INPRFM-Pax; 2009.

26. Natera G, Mora-Ríos J, Tiburcio M, Medina P. An international perspective: constructing intervention strategies for families in Mexico. Drugs: Education, Prevention and Policy. 2010;17(Suppl 1):S193-S202. doi: 10.3109/09687637.2010.514787.

27. Nuño-Gutiérrez BL, Álvarez-Nemegyei J, GonzálezForteza C, Madrigal-de León E. La adicción ¿vicio o enfermedad? Imágenes y uso de servicios de salud en adolescentes usuarios y sus padres. Salud Mental. 2006;29(4):47-54.

28. Raikhel E, Garriot W. Addiction trajectories. London: Duke University Press; 2013

29. Almanza AM. Narrativas acerca del VIH: la mirada del paciente y de su red social. Riga: Publicia; 2015.

30. Kleinman A. The illness narratives. Nueva York: Basic Books; 1988.

31. Hydén L. Illness and narrative. Sociology of Health \& Illness. 1997;19(1):48-69. doi: 10.1111/j.1467-9566. 1997.tb00015.x.

32. Good B. Medicina, racionalidad y experiencia: Una perspectiva antropológica. Barcelona: Bellaterra; 2003.

33. Gergen K. Realidades y relaciones: Aproximaciones a la construcción social. Barcelona: Paidós; 1996.

34. Frank A. The wounded storyteller: Body, illness and ethics. Chicago: The University of Chicago Press; 1995.

35. García B. El modelo Minnesota: Un método de tratamiento para las adicciones. [Tesis de maestría]. Bilbao: Universidad de Deusto; 2011.

36. Gómez-San Luis AH, Almanza-Avendaño AM. Barriers to addiction prevention and treatment in communities with organized crime: the perspective of health providers. Salud Mental. 2018;41(2):73-80. 
37. Lieblich A, Tuval R, Zilber T. Narrative research. Reading, analysis and interpretation. California: Sage; 1998.

38. Marín-Navarrete R, Eliosa-Hernández A, LozanoVerduzco I, Fernández-De la Fuente C, Turnbull B, Tena-Suck A. Estudio sobre la experiencia de hombres atendidos en centros residenciales de ayuda mutua para la atención de las adicciones. Salud Mental. 2013;36(5):393-402.

39. Harkness D, Cotrell G. The social construction of codependency in the treatment of substance abuse. Journal of Substance Abuse Treatment. 1997;14(5):473-479. doi: 10.1016/s0740-5472(97)00121-9.

40. Hänninen V, Koski-Jännes A. Narratives of recovery from addictive behaviours. Addiction. 1999;94(12):18371848. doi: 10.1046/j.1360-0443.1999.941218379.x.

41. Lozano-Verduzco I, Romero-Mendoza M, Marín-Navarrete R. Violence narratives of Mexican women treated in mutual-aid residential centers for addiction treatment. Substance Abuse Treatment, Prevention and Policy. 2016;11(1):39-49. doi: 10.1186/s13011-016-0083-0.
42. O 'Grady C, Skinner W. Journey as destination: a recovery model for families affected by concurrent disorders. Qualitative Health Research. 2012;22(8):10471062. doi: $10.1177 / 1049732312443736$.

43. Burke AC, Clapp JD. Ideology and social work practice in substance abuse settings. Social Work. 1997;42(6):552562. doi: 10.1093/sw/42.6.552.

44. Klingemann J. Lay and professional concepts of alcohol dependence in the process of recovery from addiction among treated and non-treated individuals in Poland: a qualitative study. Addiction Research \& Theory. 2011;19(3):266-275. doi: 10.3109/16066359. 2010.520771.

45. Kougiali Z, Fasulo A, Needs A, Van Laar D. Planting the seeds of change: directionality in the narrative construction of recovery from addiction. Psychology \& Health. 2017;32(6):639-664. doi: 10.1080/08870446. 2017.1293053.

\section{FORMA DE CITAR}

Almanza Avendaño AM, Gómez San Luis AH. Narrativas sobre el proceso de recuperación ante la adicción: la perspectiva de familiares que asisten a servicios de atención. Salud Colectiva. 2020;16:e2523. doi: 10.18294/sc.2020.2523.

Recibido: 31 ago 2019 | Versión final: 4 abr 2020 | Aprobado: 14 abr 2020 | Publicado en línea: 19 may 2020

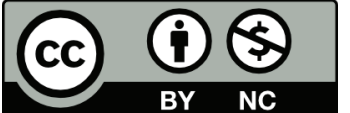

Esta obra está bajo una licencia de Creative Commons Reconocimiento-NoComercial 4.0 Internacional. Reconocimiento - Permite copiar, distribuir y comunicar públicamente la obra. A cambio, se debe reconocer y citar al autor original. No Comercial - Esta obra no puede ser utilizada con finalidades comerciales, a menos que se obtenga el permiso. 